\title{
Development of a novel rapamycin loaded nano- into micro-formulation for treatment of lung inflammation
}

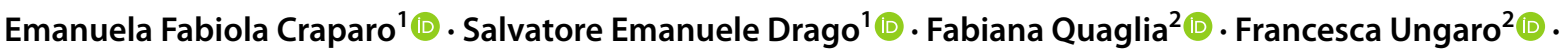 \\ Gennara Cavallaro' ${ }^{10}$
}

Accepted: 29 November 2021 / Published online: 19 February 2022

(c) The Author(s) 2022

\begin{abstract}
It has recently emerged that drugs such as the mTOR inhibitor rapamycin (Rapa) may play a key role in the treatment of airway inflammation associated with lung diseases, such as chronic obstructive pulmonary disease, asthma, and cystic fibrosis. Nevertheless, Rapa clinical application is still prevented by its unfavorable chemical-physical properties, limited oral bioavailability, and adverse effects related to non-specific biodistribution. In this paper, the design and production of a novel formulation of Rapa based on nano into micro (NiM) particles are detailed. To achieve it, Rapa-loaded nanoparticles were produced by nanoprecipitation of an amphiphilic pegylated poly- $\varepsilon$-caprolactone/polyhydroxyethyl aspartamide graft copolymer. The obtained nanoparticles that showed a drug loading of $14.4 \mathrm{wt} \%$ (corresponding to an encapsulation efficiency of $82 \mathrm{wt} \%$ ) did not interact with mucins and were able to release and protect Rapa from degradation in simulated lung and cell fluids. To allow their local administration to the lungs as a dry powder, particle engineering at micro-sized level was done by embedding nanoparticles into mannitol-based microparticles by spray drying. Obtained NiM particles had a mean diameter of about $2-\mu$, spherical shape and had good potential to be delivered to the lungs by a breath-activated dry powder inhalers. Rheological and turbidity experiments showed that these NiM particles can dissolve in lung simulated fluid and deliver the Rapa-loaded pegylated nanoparticles, which can diffuse through the mucus layer.
\end{abstract}

Keywords Pulmonary administration $\cdot$ Nanoparticles $\cdot$ Microparticles $\cdot$ Rapamycin $\cdot$ Inflammation

$\begin{array}{ll}\text { Abbreviations } & \\ \text { Rapa } & \text { Rapamycin } \\ \text { PCL nano } & \text { Empty PCL-based nanoparticles } \\ \text { Nano } & \text { Empty PHEA-g-RhB-g-SUCC- } \\ & \text { PCL-based nanoparticles } \\ \text { Rapa-loaded nano } & \text { Rapa-loaded PHEA-g-RhB-g- } \\ & \text { SUCC-PCL-based nanoparticles } \\ \text { Nano-PEG } & \text { Empty PHEA-g-RhB-g- } \\ & \text { SUCC-PCL-g-PEG-based } \\ & \text { nanoparticles }\end{array}$

Gennara Cavallaro

gennara.cavallaro@unipa.it

1 Lab of Biocompatible Polymers, Department of Biological, Chemical and Pharmaceutical Sciences and Technologies (STEBICEF), University of Palermo, Via Archirafi 32, 90123 Palermo, Italy

2 Lab of Drug Delivery, Department of Pharmacy, University of Napoli Federico II, Via D Montesano 49, 80131 Naples, Italy

$\begin{array}{ll}\text { Rapa-loaded nano-PEG } & \begin{array}{l}\text { Rapa-loaded PHEA-g-RhB- } \\ \text { g-SUCC-PCL-g-PEG-based }\end{array} \\ & \text { nanoparticles } \\ & \text { Nano- into micro-containing } \\ \text { NiM } & \text { NiMty nano } \\ & \text { nano-PEG } \\ \mathrm{NiM}_{\text {(PEG) }} & \text { NiM containing Rapa-loaded } \\ \mathrm{NiM}_{(\text {Rapa })} & \text { nano } \\ & \text { NiM containing Rapa-loaded } \\ \mathrm{NiM}_{(\text {Rapa/PEG) }} & \text { nano-PEG }\end{array}$

\section{Introduction}

Pulmonary diseases represent a major social and economic problem as many of these are chronic and require multidrug therapies $[1,2]$. Therefore, local pulmonary administration of drugs using innovative formulations with improved bioavailability and easy-to-use devices represents the main objective of many researchers in the field [3-5]. Moreover, 
the development of advanced inhalable formulations has made it possible to harness new drugs whose administration by other routes is accompanied by poor bioavailability and/or serious side effects due to non-specific biodistribution throughout the body [6, 7]. Meanwhile, innovative formulations may provide a new therapeutic opportunity for lung intervention to established drugs currently used in conventional dosage forms for the treatment of other pathologies [8-10].

Rapamycin (Rapa) is a macrolide whose major cellular target, mammalian target of rapamycin (mTOR), is one of the central regulators of growth, differentiation, metabolism, and survival in many cell types [11]. It is currently approved as an oral solution and/or film-coated tablets for the prophylaxis of organ rejection after transplant, as well as for the treatment of sporadic lymphangioleiomyomatosis (LAM). Moreover, it has been recently repurposed in the treatment of airway inflammation associated with pulmonary diseases such as chronic obstructive pulmonary disease (COPD), asthma, pulmonary arterial hypertension (PAH), and idiopathic pulmonary fibrosis (IPF) [12-14]. Very recently, Rapa is also under evaluation as a drug candidate for optimizing the treatment of coronavirusinduced disease (COVID-19) [15, 16]. Nevertheless, despite the great potential for its use in humans, Rapa applicability is severely limited by formulation problems and poor bioavailability [11].

In literature, there are already numerous attempts to formulate Rapa through innovative formulations such as nanostructured carriers with encouraging results both for the diagnosis and for the treatment of numerous pathologies [17]. Recently, some attempts to realize inhaled formulations of Rapa were reported, with interesting preliminary results $[18,19]$. These first results support the concept of local Rapa administration by inhalation to improve drug efficacy in severe lung diseases while minimizing the systemic side effects observed with oral formulations.

Once given the obvious advantage of inhalation to maximize drug bioavailability, formulation/device engineering to ensure correct deposition, drug solubilization in the lung lining fluid, and subsequent absorption is challenging. Particular attention must be given to the materials used for the realization of the aforementioned carriers, by choosing biodegradable and/or biocompatible excipients with tailored properties to control delivery features [20,21]. Mucus-penetrating nanoparticles can be achieved by decorating the carrier surfaces with suitable materials, such as polyethylene glycols (PEG), able to confer stealth properties and the ability to spread through the mucus present in the airways [22, 23]. Meanwhile, particle engineering at micro-sized level is imperative to meet the requirements allowing their local administration to the lungs as a dry powder by suitable devices, such as with breath-actuated dry powder inhalers (DPIs) [24, 25].
In this work, we develop a dry powder for Rapa inhalation through a nano-into-micro-(NiM) approach [26]. The NiM particles were obtained by spray drying and were constituted of a mannitol (Man) matrix incorporating Rapaloaded pegylated polymeric nanoparticles. The latter was produced by the nanoprecipitation method starting from a pegylated derivative of the $\alpha, \beta$-poly ( $N$-2-hydroxyethyl)-DLaspartamide (PHEA) grafted with a carboxyl-terminated PCL [27]. The properties of the developed NiM particles, in terms of aerodynamic behavior after aerosolization through a DPI and dissolution profile into a physiological medium, were evaluated. Moreover, the capability of the Rapa-loaded nanoparticles, once released due to NiM dissolution, to diffuse through a mucus layer, to protect the entrapped drug from hydrolysis into simulated lung fluid and cell medium, was also investigated.

\section{Materials and methods}

\section{Materials}

Anhydrous $N, N^{\prime}$-dimethylformamide (a-DMF), anhydrous dimethylacetamide (a-DMA), methanol, diethylether, dichloromethane, poly- $\varepsilon-$ caprolactone (PCL, $\bar{M}_{w}=10-18 \mathrm{kDa}$ ), succinic anhydride (SA), dimethylaminopyridine (DMAP), $1,1^{\prime}$-carbonyldiimidazole (CDI), $N, N^{\prime}$-disuccinimidyl carbonate (DSC), diethylamine (DEA), triethylamine (TEA), mannitol, mucin from porcine stomach (type III, bound sialic acid $0.5-1.5 \%$ ), poly(ethylene oxide) standards, O-(2-aminoethyl)-O'-methyl poly(ethylene glycol) 2000 $\left(\mathrm{H}_{2} \mathrm{~N}-\mathrm{PEG}\right)\left(\leq 0.4 \mathrm{mmol} \mathrm{NH}_{2} / \mathrm{g}, 2 \mathrm{kDa}\right)$, Dulbecco's phosphate buffer saline (DPBS), fetal bovine serum (FBS), and mannitol (Man) were of analytic grade and obtained from Sigma-Aldrich (Italy). Rapamycin (Rapa) was purchased from Accel Pharmatech (US).

${ }^{1} \mathrm{H}-\mathrm{NMR}$ spectra were registered by a Bruker Avance II-300 spectrometer, working at $300 \mathrm{MHz}$ (Bruker, Milan, Italy).

Size exclusion chromatography (SEC) analysis was performed by a system from Waters (Mildford, MA) equipped with two columns (Phenogel, 5- $\mu \mathrm{m}$ particle size, pore size: $10^{3} \AA$ and $10^{4} \AA$ ) from Phenomenex, and a refractometer. Elution parameters: $50{ }^{\circ} \mathrm{C}$, flow of $0.8 \mathrm{~mL} / \mathrm{min}$; eluent: DMF solution of $0.01 \mathrm{M} \mathrm{LiBr}$. Standards: PEGs (range $145-1.5 \mathrm{kDa})$. Sample preparation: dispersion in the eluent $(2.5 \mathrm{mg} / \mathrm{mL})$ and filtration $(0.2 \mu \mathrm{m})$. Each analysis was conducted in triplicate.

$\alpha, \beta$-Poly ( $N$-2-hydroxyethyl)-D,L-aspartamide (PHEA) and PHEA-g-RhB were properly synthesized by following procedures already reported in literature [28].

PHEA-g-RhB ${ }^{1} \mathrm{H}-\mathrm{NMR}\left(300 \mathrm{MHz}, \mathrm{D}_{2} \mathrm{O}, 25^{\circ} \mathrm{C}\right.$, TMS $): \delta$ $1.15\left(12 \mathrm{H}_{\mathrm{RhB}} \mathbf{C H}_{3} \mathrm{CH}_{2}-\right) ; \delta 2.71\left(2 \mathrm{H}_{\mathrm{PHEA}}-\mathrm{COCHCH}_{2} \mathrm{CONH}-\right)$; 
$\delta 3.29\left(2 \mathrm{H}_{\text {PHEA }}-\mathrm{NHCH}_{2} \mathrm{CH}_{2} \mathrm{O}-\right) ; \delta 3.58\left(2 \mathrm{H}_{\text {PHEA }}-\mathrm{NHCH}-\right.$ $\left.{ }_{2} \mathbf{C H}_{2} \mathrm{O}-\right) ; \delta 4.65\left(1 \mathrm{H}_{\mathrm{PHEA}}-\mathrm{NHCH}(\mathrm{CO}) \mathrm{CH}_{2}-\right) ; \delta 8.00-8.50$ $\left(10 \mathrm{H}_{\mathrm{RhB}} \mathbf{H}-\mathrm{Ar}\right)$. The $\bar{M}_{w}$ of PHEA-g-RhB used in this study was $52.5 \mathrm{Da}\left(\bar{M}_{w} / \bar{M}_{n}=1.6\right)$. The degree of derivatization in $\mathrm{RhB}$ $\left(\mathrm{DD}_{\mathrm{RhB}}\right)$, determined from the ${ }^{1} \mathrm{H}-\mathrm{NMR}$ spectra, as reported elsewhere, was equal to $0.6 \pm 0.05 \mathrm{~mol} \%$ [29].

Poly- $\varepsilon$-caprolactone-succinate (SUCC-PCL) was synthesized and characterized as reported elsewhere [27, 30].

\section{Synthesis and characterization of PHEA-g-RhB-g-SUCC-PCL graft copolymer}

A modified synthetic procedure was followed to synthesize PHEA-g-RhB-g-SUCC-PCL graft copolymer with proper derivatization degree (DD\%) [30]. Briefly, to an organic PHEA-g-RhB dispersion in a-DMF $(33 \mathrm{mg} / \mathrm{mL})$, DEA was added as catalyst according to $R_{2}=0.3$ that is the molar ratio between DEA and those of repeating units (RUs) of PHEA-g-RhB carrying hydroxyl groups. At the same time, on the basis of $R_{1}=3$ (that is the mole ratio between CDI and PCL-SUCC), the calculated amount of CDI was added to the organic PCL-SUCC dispersion $(66.5 \mathrm{mg} / \mathrm{mL}$ in a-DMF), and the resulting mixture was putted at $40{ }^{\circ} \mathrm{C}$ for $5 \mathrm{~h}$. After this time, the PHEA-RhB dispersion was added dropwise to that of CDI-activated PCL-SUCC according to the molar ratio between PCLSUCC and those of PHEA-g-RhB RUs equal to $R_{3}=0.12$. The resulting mixture was left at $40{ }^{\circ} \mathrm{C}$ for $68 \mathrm{~h}$, then the product was recovered by precipitation in diethyl ether, separated from the supernatant by centrifugation (at $4{ }^{\circ} \mathrm{C}$ for $15 \mathrm{~min}$, at $9800 \mathrm{rpm}$ ), and washed three times with a diethylether:dichloromethane mixture $(4: 1 \mathrm{v} / \mathrm{v})$. The obtained product was dissolved in DMA, purified by dialysis against water (MWCO 12-14 kDa), and freeze-dried.

${ }^{1} \mathrm{H}-\mathrm{NMR}$ (300 MHz, [D7].DMF, $25{ }^{\circ} \mathrm{C}$, TMS): $\delta 1.13$ $\left(m, 12 \mathrm{H}_{\mathrm{RhB}} \mathbf{C H}_{3} \mathrm{CH}_{2}-\right) ; \delta 1.5$ and $2.1\left(m, 6 \mathrm{H}_{\mathrm{PCL}}-[\mathrm{O}(\mathrm{O})\right.$ $\left.\left.\mathrm{CCH}_{2}\left(\mathbf{C H}_{2}\right)_{3} \mathrm{CH}_{2}\right]_{122}\right) ; \delta 2.5\left(2 \mathrm{~d}, 2 \mathrm{H}_{\mathrm{PCL}}-\left[\mathrm{O}(\mathrm{O}) \mathrm{CCH}_{2}\right.\right.$ $\left.\left.\left(\mathrm{CH}_{2}\right)_{3} \mathbf{C H}_{2}\right]_{122}-\right) ; \delta 2.8\left(m, 2 \mathrm{H}_{\mathrm{PHEA}}-\mathrm{C}(\mathrm{O}) \mathrm{CHCH}_{2} \mathrm{C}(\mathrm{O})\right.$ $\mathrm{NH}-) ; \delta 3.2\left(t, 2 \mathrm{H}_{\mathrm{PHEA}}-\mathrm{NHCH}_{2} \mathrm{CH}_{2} \mathrm{O}-\right) ; \delta 3.50(t$, $\left.2 \mathrm{H}_{\mathrm{PHEA}}-\mathrm{NHCH}_{2} \mathbf{C H}_{\mathbf{2}} \mathrm{O}-\right) ; \delta 4.3\left(t, 2 \mathrm{H}_{\mathrm{PCL}}-\left[\mathrm{O}(\mathrm{O}) \mathrm{CCH}_{\mathbf{2}}(\mathrm{C}\right.\right.$ $\left.\left.\mathrm{H}_{2}\right)_{3} \mathrm{CH}_{2}\right]_{122}-$ ), and $\delta 5.0\left(m, 1 \mathrm{H}_{\mathrm{PHEA}}-\mathrm{NHCH}(\mathrm{CO}) \mathrm{CH}_{2}-\right)$; $\delta 7.00-8.00\left(m, 10 \mathrm{H}_{\mathrm{RhB}}\right.$ H-Ar $)$.

\section{PEGylation of PHEA-g-RhB-g-SUCC-PCL graft copolymer}

PEGylation of PHEA-g-RhB-g-SUCC-PCL to obtain PHEA-g-RhB-g-SUCC-PCL-g-PEG graft copolymer was done as already reported for similar copolymers [27, 31]. Briefly, to an organic a-DMA dispersion of PHEAg-RhB-g-SUCC-PCL (64 mg/mL), TEA as catalyst, and DSC were added according to $R_{4}=0.1$ (the molar ratio between DSC and moles of PHEA RUs carrying hydroxyl groups), and $R_{5}=1$ (the molar ratio between TEA and moles of DSC). The obtained dispersion was placed to reach at $40{ }^{\circ} \mathrm{C}$. After $4 \mathrm{~h}$, the latter was added dropwise to an organic a-DMA dispersion of $\mathrm{H}_{2} \mathrm{~N}-\mathrm{PEG}(12 \mathrm{mg} / \mathrm{mL})$, according to $R_{6}=0.075$, being $R_{6}$ the molar ratio between $\mathrm{H}_{2} \mathrm{~N}$-PEG and moles of PHEA-g-RhB-g-SUCC-PCL RUs carrying hydroxyl groups. After $18 \mathrm{~h}$ at $25^{\circ} \mathrm{C}$, the reaction mixture was purified by dialysis (MWCO 12-14 kDa) against distilled water and freeze-dried to recover the obtained copolymer. PHEA-g-RhB-g-SUCC-PCL-g-PEG graft copolymer was obtained with a yield of $240 \mathrm{wt} \%$ based on the starting PHEA-g-RhB-g-SUCC-PCL.

${ }^{1} \mathrm{H}-\mathrm{NMR}$ (300 MHz, [D7].DMF, $25{ }^{\circ} \mathrm{C}$, TMS): $\delta 1.13$ $\left(m, 12 \mathrm{H}_{\mathrm{RhB}} \mathbf{C H}_{3} \mathrm{CH}_{2}-\right) ; \delta 1.5$ and $2.1\left(m, 6 \mathrm{H}_{\mathrm{PCL}}-[\mathrm{O}(\mathrm{O})\right.$ $\left.\left.\mathrm{CCH}_{2}\left(\mathbf{C H}_{2}\right)_{3} \mathrm{CH}_{2}\right]_{122}-\right) ; \delta 2.5\left(2 \mathrm{~d}, 2 \mathrm{H}_{\mathrm{PCL}}-\left[\mathrm{O}(\mathrm{O}) \mathrm{CCH}_{2}(\mathrm{C}\right.\right.$ $\left.\left.\left.\mathrm{H}_{2}\right)_{3} \mathbf{C H}_{2}\right]_{122}\right) ; \delta 2.8\left(m, 2 \mathrm{H}_{\mathrm{PHEA}}-\mathrm{C}(\mathrm{O}) \mathrm{CHCH}_{2} \mathrm{C}(\mathrm{O}) \mathrm{NH}-\right)$; $\delta 3.2\left(t, 2 \mathrm{H}_{\text {PHEA }}-\mathrm{NHCH}_{2} \mathrm{CH}_{2} \mathrm{O}-\right) ; \delta 3.50\left(t, 2 \mathrm{H}_{\text {PHEA }}\right.$ $\left.-\mathrm{NHCH}_{2} \mathbf{C H}_{2} \mathrm{O}-\right) ; \delta 3.7\left(t, 4 \mathrm{H}_{\mathrm{PEG}}-\left[\mathrm{CH}_{2} \mathbf{C H}_{2} \mathrm{O}\right]_{44^{-}}\right) ; \delta$ $4.3\left(t, 2 \mathrm{H}_{\mathrm{PCL}}-\left[\mathrm{O}(\mathrm{O}) \mathrm{CCH}_{2}\left(\mathrm{CH}_{2}\right)_{3} \mathrm{CH}_{2}\right]_{122}-\right)$; and $\delta 5.0$ $\left(m, 1 \mathrm{H}_{\mathrm{PHEA}}-\mathrm{NHCH}(\mathrm{CO}) \mathrm{CH}_{2}-\right) ; \delta 7.00-8.00\left(m, 10 \mathrm{H}_{\mathrm{RhB}}\right.$ H-Ar).

\section{Nanoparticle production}

Empty or Rapa-loaded pegylated nanoparticles (empty nano-PEG and Rapa-loaded nano-PEG, respectively) were obtained by nano-precipitation. In particular, a PHEA-gRhB-g-SUCC-PCL-g-PEG graft copolymer dispersion of $(1.5 \% \mathrm{w} / \mathrm{v})$ in DMA (containing or not the drug at a concentration of $0.32 \% \mathrm{w} / \mathrm{v}$ ) was placed in a burette and added dropwise to twice-distilled water $(1: 10 \mathrm{v} / \mathrm{v})$. The mixture was left under stirring for $2 \mathrm{~h}$, dialyzed against twicedistilled water, centrifuged, and filtered, and the obtained nanoparticle dispersion was stored at $5{ }^{\circ} \mathrm{C}$ before being used or for further characterization. To obtain empty and drugloaded non-pegylated nanoparticles (empty and Rapa-loaded Nano), the procedure of nanoprecipitation was followed by using PHEA-g-RhB-g-SUCC-PCL graft copolymer. To obtain PCL-based nanoparticles (PCL nano), the same procedure was followed by using PCL as starting polymeric material.

\section{Nanoparticle characterization}

Size and $\zeta$ potential measurements Hydrodynamic diameter ( $Z$-average), polydispersity index (PDI), and $\zeta$ potential of each sample were determined by using a Malvern Zetasizer Nano ZSP instrument (Malvern Instrument, Malvern, $\mathrm{UK}), \mathrm{a} \mathrm{He}-\mathrm{Ne}$ laser at $\lambda=632.8 \mathrm{~nm}$, and at a fixed scattering angle of $175^{\circ}$. Each sample, freshly prepared or dispersed in ultrapure water, was analyzed at $25^{\circ} \mathrm{C}$ [30]. Each measurement was repeated in triplicate. 
XPS analysis A PHI 5000 VersaProbe II (ULVAC-PHI, Inc.) was used to record XPS spectra of each sample, by using a monochromatic Al-K $\alpha$ radiation $(h \nu=1486.6 \mathrm{eV})$ from an $\mathrm{X}$-ray source operating at a spot size of $200 \mu \mathrm{m}$, a power of $50 \mathrm{~W}$ and an acceleration voltage of $15 \mathrm{kV}$.

Determination of drug loading The drug loading (DL\%), that is the Rapa amount loaded into each drug-loaded sample (nano-PEG or nano), and entrapment efficiency (EE\%), expressed as the weight percent ratio between the amount of Rapa actually entrapped into the particles and the theoretical one, were assessed by HPLC analyses. In detail, a Waters Breeze System Liquid Chromatograph system was used, which was equipped with a Luna ${ }^{\circledR} \mathrm{C} 18$ column $(250 \times 4.6 \mathrm{~mm}, 5 \mu \mathrm{m}$, from Phenomenex), an autosampler (40 $\mu \mathrm{L}$ as injected volume), and an UV - vis HPLC detector. Other parameters are as follows: a methanol:water $80: 20 \mathrm{v} / \mathrm{v}$ solution as mobile phase, a flow rate of $1 \mathrm{~mL} / \mathrm{min}$, temperature equal to $25{ }^{\circ} \mathrm{C}$, detection wavelength equal to $277 \mathrm{~nm}$. By plotting peak areas (at retention time $=14 \mathrm{~min}$ ) versus Rapa standard concentration values in methanol (range of $0.02-0.001 \mathrm{mg} / \mathrm{mL}$ ), a calibration curve was built $\left(y=136,513 x, R^{2}=0.9994\right)$. Each sample was treated as follows: a proper amount was dissolved in DMA, added with methanol (1:9 v/v, $2.5 \mathrm{mg} / \mathrm{mL})$ and the obtained dispersion filtered (with filters at a $0.45-\mu \mathrm{m}$ pore size). The resulting solution was analyzed by HPLC. Each obtained peak area at 14 min was compared with the calibration curve.

\section{Cell viability assay}

Cell viability was assessed by a 3-(4,5-dimethylthiazol2-yl)-5-(3-carboxymethoxyphenyl)-2-(4-sulphophenyl)$2 \mathrm{H}$-tetrazolium (MTS) assay on 16HBE cells, using a commercially available kit (Cell Titer 96 Aqueous One Solution Cell Proliferation assay, Promega) containing MTS and phenazine ethosulfate. 16HBE cells were plated on a 96-well plate at a cell density of 15,000 cells/well in DMEM containing $10 \%$ FBS. After $24 \mathrm{~h}$ of incubation, the medium was removed, and then, the cells were incubated with $200 \mu \mathrm{L}$ per well with an aqueous dispersion (DMEM containing 10\% FBS) of empty nano-PEG or Rapa-loaded nano-PEG (at concentrations ranging between 0.05 and $0.75 \mathrm{mg} / \mathrm{mL}$ ). Cell viability in the presence of free Rapa, at concentrations corresponding to those loaded into the Rapa-loaded Nano-PEG sample, was also evaluated. All dispersions were sterilized by filtration using 220-nm filter. After 24- and 48-h incubation, supernatant was removed and each plate was washed with sterile DPBS; after this, cells in each well were incubated with $100 \mu \mathrm{L}$ of fresh DMEM and $20 \mu \mathrm{L}$ of a MTS solution, and plates were incubated for $2 \mathrm{~h}$ at $37^{\circ} \mathrm{C}$. The absorbance at $490 \mathrm{~nm}$ was read using a microplate reader (Multiskan Ex, Thermo
Labsystems, Finland). Relative cell viability (percentage) was expressed as (Abs490 treated cells/Abs490 control cells) $\times 100$, based on three experiments. Cells incubated with the medium were used as negative control.

\section{Production of NiM particles}

$\mathrm{NiM}$ formulations were obtained by following a previously reported procedure [26]. In detail, the spray drying process was carried out by using a Buchi Nano Spray Dryer B-90. Liquid feed aqueous dispersions containing Rapa-loaded nano-PEG or Rapa-loaded nano $(0.75 \mathrm{mg} / \mathrm{mL})$ and Man $(1 \mathrm{~g} / 100 \mathrm{~mL})$ (nanoparticles/Man 1:13 weight ratio) were used to obtain, respectively, $\mathrm{NiM}_{(\mathrm{Rapa} / \mathrm{PEG})}$ or $\mathrm{NiM}_{(\text {Rapa) }}$ samples. Before use, each dispersion was sorted through a 1.2$\mu \mathrm{m}$ filter and then spray-dried with a large spray nebulizer at the inlet temperature of $100{ }^{\circ} \mathrm{C}$. Filtered and dehumidified air was used as the drying gas; the drying gas flow rate was $120 \mathrm{~L} / \mathrm{min}$ resulting in an inside pressure of $27 \mathrm{mbar}$ with a spray rate of $78 \%$ and pump $66 \%$. Each collected NiM sample was appropriately stored at $-20{ }^{\circ} \mathrm{C}$ before analysis.

The amount of Rapa loaded into each NiM sample was determined by HPLC. In detail, a known amount of NiM was dissolved in a mixture of DMA and methanol $(2.5 \mathrm{mg} /$ $\mathrm{mL}$ ) for almost $2 \mathrm{~h}$, the obtained dispersion was filtered $(0.45 \mu \mathrm{m})$, and the supernatant was analyzed by HPLC following the method reported above.

\section{NiM characterization}

SEM and OM analyses $\mathrm{NiM}_{\text {(Rapa/PEG) }}$ sample was laid on a double-sided adhesive tape, previously applied on a stainless steel stub, which was then sputter-coated with gold prior to microscopy examination, and then observed by using by using a Phenom ${ }^{\mathrm{TM}}$ ProX Desktop SEM microscope.

The OM analysis was conducted by recording transmittance images of $\mathrm{NiM}_{(\mathrm{Rap} / \mathrm{PEG})}$ dispersed in paraffin oil with a ZEISS optical microscope, using the AxioVision software.

The ImageJ program was used to calculate the average diameter of each sample from either SEM or OM images by analyzing a sufficiently representative number to constitute a certain datum ( $>500$ particles).

Drug stability The Rapa stability was evaluated in physiologic conditions mimicking fluid by quantifying the amount of intact drug over time. In particular, a known amount of Rapa (0.09 mg), free or loaded into $\mathrm{NiM}_{\text {(Rapa/PEG) }}$, was dispersed in $30 \mathrm{~mL}$ of simulated lung fluid (SLF4) $\left(\mathrm{MgCl}_{2}\right.$ $(0,2033 \mathrm{~g} / \mathrm{L}), \mathrm{NaCl}(6.0193 \mathrm{~g} / \mathrm{L}), \mathrm{KCl}(0.2982 \mathrm{~g} / \mathrm{L})$, $\mathrm{Na}_{2} \mathrm{SO}_{4}(0.0710), \mathrm{CaCl}_{2}$ dihydrate $(0,3676 \mathrm{~g} / \mathrm{L})$, sodium acetate $(0.9526 \mathrm{~g} / \mathrm{L}), \mathrm{NaHCO}_{3}$ (2.6043), sodium citrate dihydrate $(0.0970 \mathrm{~g} / \mathrm{L}), \mathrm{NaH}_{2} \mathrm{PO}_{4}$ monohydrate $(0.1420)$, dipalmitoylphosphatidylcholine (DPPC, $0,02 \mathrm{w} / \mathrm{v} \%)$ ), 
or cell medium (Dulbecco's phosphate-buffered saline (DPBS):fetal bovine serum (FBS) $(90: 10 \mathrm{v} / \mathrm{v})$ mixture) [32]. At fixed times $(0,1,2,4,7,12,16$, and $24 \mathrm{~h})$, each dispersion was freeze-dried and properly treated in order to recover the intact drug. For free Rapa stability, each freezedried sample was treated with $7 \mathrm{~mL}$ of methanol, while for Rapa-loaded $\mathrm{NiM}_{(\mathrm{Rapa} / \mathrm{PEG})}$, the freeze-dried sample was treated with $2 \mathrm{~mL}$ of DMA and $5 \mathrm{~mL}$ of methanol. Then, the obtained organic dispersions were centrifuged and the supernatants were analyzed by HPLC.

Drug release The Rapa release profile was evaluated in physiologic conditions mimicking fluid by quantifying the amount of intact drug released from the sample over time. In particular, a known amount of Rapa $(0.09 \mathrm{mg})$ loaded into $\mathrm{NiM}_{(\text {Rapa/PEG) }}$, was dispersed in $30 \mathrm{~mL}$ of simulated lung fluid (SLF4), or cell medium [32]. At fixed times (0, 1, 2, 4, $7,12,16$, and $24 \mathrm{~h}$ ), each dispersion was ultra-centrifuged; the supernatant was freeze-dried and properly treated in order to recover the intact drug. In particular, the freezedried sample was treated with $1.5 \mathrm{~mL}$ of methanol. Then, the obtained organic dispersions were centrifuged, and the supernatants were analyzed by HPLC.

\section{NIM aerodynamic behavior}

The aerosolization properties of $\mathrm{NiM}_{(\mathrm{Rapa} / \mathrm{PEG})}$ were tested after delivery from breath-activated reusable DPIs working with single unit capsule containing the dry powder using a next-generation impactor (NGI) (Copley Scientific, UK) according to Ph. Eur. 10th Ed. Two devices with different resistances to the airflow were tested: the low-resistance DPI RS01 (Plastiape, Italy) and the medium-resistance DPI TurboSpin ${ }^{\circledR}$ (PH\&T Pharma, Italy. For each test, a hard gelatin capsule (size 2, Capsugel, USA) was filled with about $20 \mathrm{mg}$ of the powder and placed in the DPI. The NGI was activated at 60 or $90 \mathrm{~L} / \mathrm{min}$, respectively, for TurboSpin ${ }^{\circledR}$ and RS01.

The powder deposited on the seven NGI collection cups, in the induction port and in the micro-orifice collector (MOC), was quantitatively recovered by dissolution in an appropriate amount of DMF. The amount of NiM in the samples was determined by spectrofluorimetric analysis at $\lambda_{\mathrm{ex}}=520 \mathrm{~nm}$. A calibration curve was derived from serial dilutions of a standard solution of fluorescent NiM $(2.5 \mathrm{mg} /$ $\mathrm{mL})$ in DMF $(0.0125-1.25 \mathrm{mg} / \mathrm{mL}$ concentration range, $R^{2} \geq 0.99$ ).

The experimental mass median aerodynamic diameter $\left(\mathrm{MMAD}_{\exp }\right)$ was calculated according to Ph.Eur. deriving a plot of cumulative mass of powder deposited in each collection cup versus cut-off diameter of the respective stage. The fine particle fraction (FPF) was calculated considering the actual amount of NiMs deposited on stages with
MMAD $<5 \mu \mathrm{m}$ as compared to the initial amount loaded into the DPI (nominal dose of NiMs). The respirable fraction (RF) was defined as the percentage of NiMs deposited on stages with MMAD $<5 \mu \mathrm{m}$ as compared to the total dose of NiMs deposited in the NGI.

\section{Interaction with artificial mucus}

Rheological analysis Measurements of interactions between each chosen sample and mucin were determined by rheological analysis at the temperature of $37{ }^{\circ} \mathrm{C}$ by using a rheometer (TA Instruments) equipped with concentric cylinders geometry. A strain sweep (5-30\%) was performed on mucin dispersion at $1.0 \mathrm{~Hz}$ to determine the linear viscoelastic region, which was found to be in the range of $10-20 \%$. Then, a time sweep (30 min) was performed for all samples at $15 \%$ constant strain and $1.0 \mathrm{~Hz}$ constant frequency to determine complex viscosity $\left(\eta^{*}\right)$. Chosen samples were as follows: $\mathrm{NiM}_{\text {(Rapa/PEG) }}$ (corresponding to $14 \mathrm{mg}$ of Rapa-loaded nano-PEG), $\mathrm{NiM}_{\text {(Rapa) }}$ (corresponding to $14 \mathrm{mg}$ of Rapaloaded nano), free Rapa (at a concentration corresponding to the drug loaded into the nanoparticles), and chitosan as positive control. For the analyses of mucin-sample mixture, a certain amount of each sample was added to $14 \mathrm{~mL}$ of mucin dispersion in PBS $(1 \mathrm{mg} / \mathrm{mL})$ and mixed gently with a spatula for $20 \mathrm{~s}$. Obtained dispersion was loaded in the rheometer and then equilibrated to $37{ }^{\circ} \mathrm{C}$ for $20 \mathrm{~min}$. To prevent dehydration during rheological measurements, a solvent trap was placed on the top of the geometry.

Turbidimetric analysis Measurements of interactions between nanoparticles and mucin were determined by turbidimetry. A proper amount of NiM, corresponding to $0.2 \mathrm{mg}$ of NanoPEG were dispersed in $190 \mu \mathrm{L}$ of PBS, were mixed with $10 \mu \mathrm{L}$ of mucin dispersion at the concentration of $20 \mathrm{mg} / \mathrm{mL}$ in PBS. Analyzed samples were as follows: $\mathrm{NiM}_{(\text {Rapa/PEG) }}, \mathrm{NiM}_{\text {(Rapa) }}$, and free Rapa (at the concentration corresponding to the drug loaded into the nanoparticles). After incubation at $37^{\circ} \mathrm{C}$, the turbidity was measured each 50 min until $6 \mathrm{~h}$. The transmittance at the $\lambda$ of $570 \mathrm{~nm}$ was recorded by microplate reader (Multiskan Ex, Thermo Labsystems, Finland). The value obtained from a mucin-free dispersion of each sample was subtracted from each transmittance value. Data were expressed as a percentage ratio between the transmittance of the sample and the transmittance of the $1 \mathrm{mg} / \mathrm{mL}$ mucin dispersion.

Muco-diffusion assay The capability of the Nano-PEG to diffuse through a mucin dispersion was evaluated using a diffusion test, as reported elsewhere [26]. Sixty milligrams of agarose was dispersed in $20 \mathrm{~mL}$ of distilled water, and the dispersion was heated until a clear solution was obtained; $1.3 \mathrm{~mL}$ of this dispersion was deposited in several 4-mL vials, allowed to harden at room temperature and stored 
at $4{ }^{\circ} \mathrm{C}$ until use. Subsequently, $2 \mathrm{~mL}$ of mucin dispersion $(1 \mathrm{mg} / \mathrm{mL}$ in PBS$)$ was placed on the hardened agarose gel, and $600 \mu \mathrm{L}$ of a $\mathrm{NiM}_{\text {(Rapa/PEG) }}$ or $\mathrm{NiM}_{\text {(Rapa) }}$ aqueous dispersion $(5 \mathrm{mg} / \mathrm{mL})$ was placed on the mucin layer and incubated at $37{ }^{\circ} \mathrm{C}$. At regular time intervals $(2,4,6,8$, and $24 \mathrm{~h}$ ), the mucin layer containing each sample was removed; the remaining agarose gels were rinsed three times with $2 \mathrm{~mL}$ of distilled water, dissolved at $60^{\circ} \mathrm{C}$, and analyzed by UV spectrophotometry, at the $\lambda$ of $561 \mathrm{~nm}$.

\section{Statistical analysis}

All the experiments were repeated at least three times. All data are expressed as means \pm standard deviation. All data were analyzed by Student's $t$ test. A $p$-value $<0.05$ was considered statistically significant, while a $p$-value $<0.01$ was considered highly significant.

\section{Results and discussion}

In this paper, a novel inhalable formulation for repositioning of rapamycin (Rapa) was developed for the management of inflammation-based lung diseases such as asthma and chronic obstructive pulmonary disease (COPD). In particular, a powder product was obtained by following the nano-into-micro-(NiM) approach, which comprises the incorporation of Rapa-loaded polymeric nanoparticles into sugar-based microparticles to be administered by dry powder inhalers (DPIs). Upon inhalation, these microparticles can dissolve releasing the nanostructured carriers, which will protect and deliver the drug cargo through the mucus layer until the bronchial epithelium.

\section{Design and development of polymeric nanostructured carriers for Rapa}

To realize the nanostructured carrier, a polymeric material with proper functional and structural properties suitable for pulmonary administration was designed and synthesized by a modification of a synthetic procedure already described [27]. In particular, on the $\alpha, \beta$-poly $(N-2$-hydroxyethyl)-DLaspartamide (PHEA), rhodamine B (RhB), the succinylated derivative of poly- $\varepsilon$-caprolactone (PCL-SUCC), and the polyethylene glycol (PEG), were sequentially grafted, obtaining the PHEA-g-RhB-g-SUCC-PCL-PEG. To design nanoparticles suited for inhalation, each component was properly chosen. PHEA, the main protein-like polymer chain, was chosen due to its biocompatibility, essential for pulmonary administration, and for the fact that offers the opportunity to graft various functionalities due to the presence of a reactive group on each repeating unit $[29,33]$. The $\mathrm{RhB}$ was chosen as fluorescent dye and covalently linked on the PHEA backbone to make it stably fluorescent without modifying its self-assembly characteristics, making it detectable and quantifiable by spectroscopic techniques [34]. PCL-SUCC was grafted on the PHEA-g-RhB to endow the resulting copolymer with suitable amphiphilic properties and to make it an excellent starting material for the nanoparticle production, able to entrap hydrophobic drugs such as Rapa. The PEG was chosen as a further material to be grafted on the PHEA backbone to increase the hydrophilicity of the nanoparticle surface, thus giving stealth properties and the ability to spread through the mucus present in the airways [22, 23]. Last but not least, all the selected polymeric materials represent valid candidates to be used for the realization of safe carriers for inhalation as their in vitro-in vivo biocompatibility has already been demonstrated [27, 29]. The synthetic steps and chemical structure of the PHEA-g-RhB-g-SUCCPCL-g-PEG graft copolymer are reported in Fig. 1.

The degree of derivatization in $\mathrm{RhB}\left(\mathrm{DD}_{\mathrm{RhB}}\right)$, PCL $\left(D_{\mathrm{PCL}}\right)$, and PEG $\left(\mathrm{DD}_{\mathrm{PEG}}\right)$ was determined from the ${ }^{1} \mathrm{H}$ NMR spectra, as reported elsewhere, and result to be equal to $0.6 \pm 0.05 \mathrm{~mol} \%, 3.4 \pm 0.1 \mathrm{~mol} \%$, and $4.3 \pm 0.06 \mathrm{~mol} \%$, respectively [27, 31]. Characterization by size exclusion chromatography (SEC) confirmed the occurrence of the conjugation reactions, being the $\bar{M}_{w}$ equal to $182 \mathrm{kDa}$ $\left(\bar{M}_{w} / \bar{M}_{n}=1.28\right)$.

Then, starting from the PHEA-g-RhB-g-SUCC-PCLg-PEG graft copolymer, the nanoprecipitation method was followed to obtain polymeric pegylated nanoparticles (empty nano-PEG). Experimental parameters and condition to carry out the nanoprecipitation are reported in detail in the experimental part.

Freshly prepared nanoparticle dispersions underwent size and surface analyses. Both empty and Rapa-loaded nano-PEG were smaller than $100 \mathrm{~nm}$, with a negative לpotential (Table 1).

As previously stated, the choice to graft PEG chains on the starting copolymer stems from the idea that the nanoprecipitation could produce nanoparticulate systems with PEG chains exposed on the surface, which could favor the diffusion through the mucus layer. Therefore, the surface chemical composition of PHEA-g-RhB-gSUCC-PCL-g-PEG-based nanoparticles (named empty nano-PEG sample) was analyzed to evaluate the surface pegylation through the X-ray photoelectron spectroscopy (XPS) technique. To better highlight the presence of PEG on the surface of the nano-PEG particles and the extent of this pegylation, the XPS analysis was also carried out on nanoparticles obtained starting from PCL alone (named empty PCL nanosample), or from PHEA-g-RhB-g-SUCCPCL copolymer (named empty nano sample).

The XPS analysis of the investigated samples is reported in Table 2 as the relative distribution of the carbon, oxygen, 
Fig. 1 The synthetic route of PHEA-g-RhB-g-SUCCPCL-g-PEG graft copolymer $(n=122, m=44)$. Reagents and conditions: (step 1) a-DMF, CDI, DEA, $4 \mathrm{~h}$ at $40{ }^{\circ} \mathrm{C}, 48 \mathrm{~h}$ at $40{ }^{\circ} \mathrm{C}$; (step 2) a-DMF, CDI, DEA, $5 \mathrm{~h}$ at $40^{\circ} \mathrm{C}, 68 \mathrm{~h}$ at $40{ }^{\circ} \mathrm{C}$; (step 3) a-DMA, DSC, TEA, $4 \mathrm{~h}$ at $40{ }^{\circ} \mathrm{C}, 18 \mathrm{~h} \mathrm{rt}$

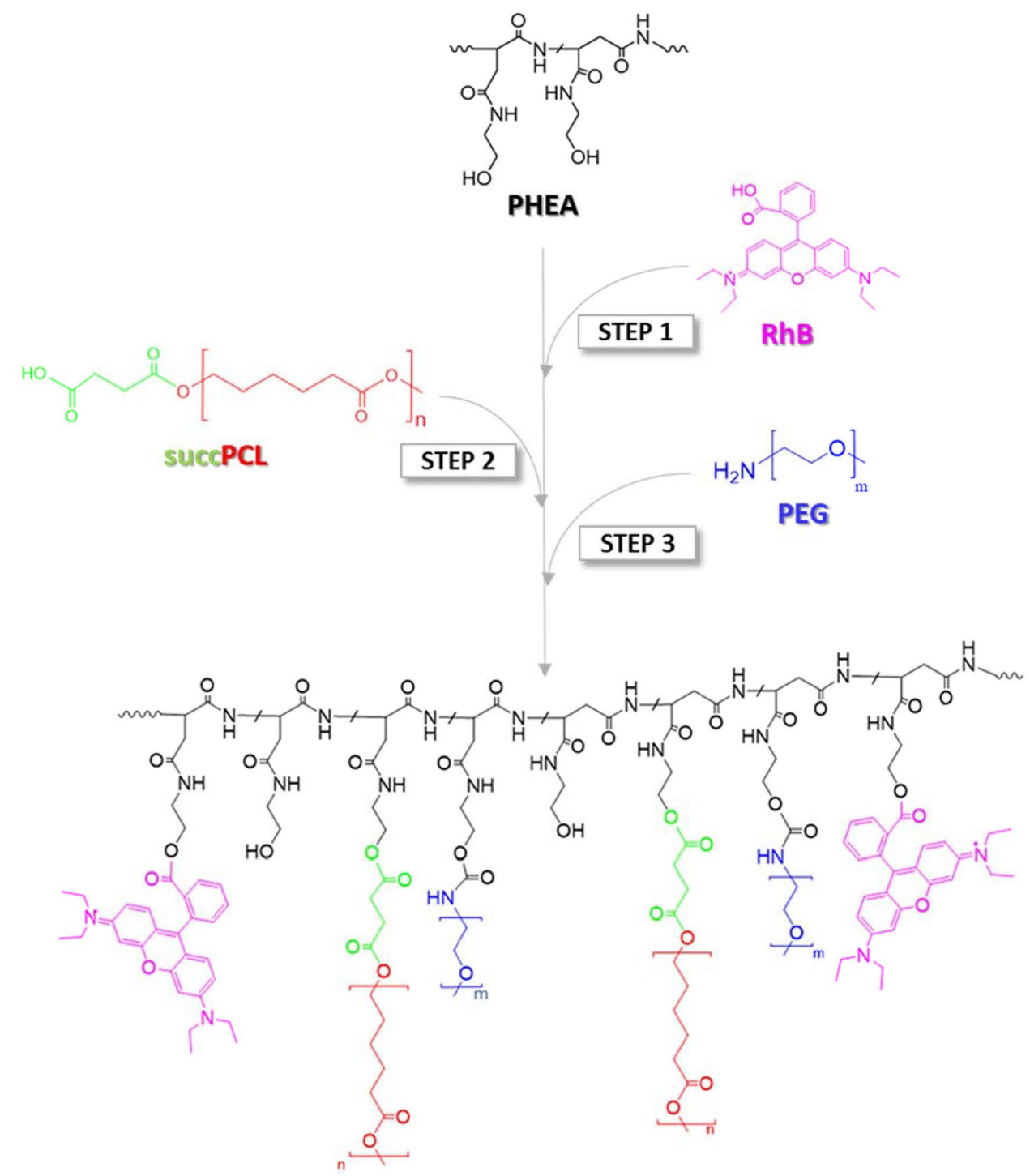

PHEA-g-RhB-g-sUCCPCL-g-PEG and nitrogen species on the particle surface, determined by a curve-fitting procedure of the photoelectron peaks. It is evident that the $\mathrm{C} 1 \mathrm{~s}$ relative atomic percentage on the empty PCL nano sample is higher than that found on empty nano and empty nano-PEG samples, that are both PHEA derivative-based nanoparticles, where instead it is present nitrogen, at binding energy equal to $399.7 \mathrm{eV}$. It

Table 1 Mean size, polydispersity index (PDI), and $\zeta$-potential of freshly prepared empty and Rapa-loaded nano-PEG in bidistilled water

\begin{tabular}{llll}
\hline Sample & $Z$-average $(\mathbf{n m})$ & PDI & $\begin{array}{c}\zeta \text { potential } \\
(\mathbf{m V} \pm \mathbf{S D})\end{array}$ \\
\hline Empty nano-PEG & 54.7 & 0.34 & $-16.7 \pm 7.8$ \\
Rapa-loaded nano-PEG & 51.1 & 0.20 & $-14.4 \pm 4.6$ \\
\hline
\end{tabular}

should be attributed to $\mathrm{N}-\mathrm{C} / \mathrm{N}-\mathrm{C}(\mathrm{O})$ linkage present in the PHEA backbone and, as expected, is absent in the PCLbased sample (empty PCL Nano). This result indicates that on the empty nano and empty nano-PEG sample surface is exposed PHEA backbone [27].

To obtain more detailed information on the type of bond that affects carbon, the relative distributions of the carbon species on the nanoparticle surface were determined by a curve-fitting procedure, and results are reported in Table 3.

As expected, in both the empty nano and empty nanoPEG samples, the $\mathrm{C} 1 \mathrm{~s}$ core level spectrum showed species at the binding energy (BE) of 286.0 and $287.6 \mathrm{eV}$, attributed respectively to $\mathrm{C}-\mathrm{N}$ and $\mathrm{N}-\mathrm{C}=\mathrm{O}$ bonds (belonging to PHEA backbone), that are absent in the empty PCL-based sample. Very interestingly, the $\mathrm{C} 1 \mathrm{~s}$ core level spectrum at the $\mathrm{BE}$ of $286.2 \mathrm{eV}$, is more than double in the empty 
Table 2 XPS Surface chemical composition of obtained nanoparticles ${ }^{\mathrm{a}}$

\begin{tabular}{llll}
\hline Nanoparticle sample & C 1 s & O 1 s & N 1 s \\
\hline Empty PCL nano $^{\mathbf{b}}$ & 74.49 & 25.51 & -- \\
Empty nano & 69.70 & 26.40 & 3.90 \\
Empty nano-PEG & 72.60 & 25.32 & 2.08 \\
\hline
\end{tabular}

${ }^{a}$ Relative distribution of the carbon, nitrogen, oxygen, and phosphorus species on the nanoparticle surface determined by a curve-fitting procedure of the photoelectron peaks

${ }^{\mathrm{b}}$ Nano obtained from PCL alone by nanoprecipitation

nano-PEG than in the other samples (42.9 vs. $16.6 \%$ ); since this level is assigned to $\mathrm{C}-\mathrm{O}-\mathrm{C}$ bonds belonging to PEG chains, it demonstrates the presence of PEG on these nanoparticles [26].

In Fig. 2( $a$ and $b$ ), the curve fittings of the $\mathrm{C} 1 \mathrm{~s}$ spectra of empty nano and nano-PEG samples are reported, respectively. As expected, in these graphics, the $\mathrm{C} 1 \mathrm{~s}$ core level spectrum at the BE of $286.2 \mathrm{eV}$ of the nano-PEG was significantly higher and mainly represented than that found on the spectrum of nano.

From the characterization of empty systems, it emerged that nano-PEG particles are of adequate size and expose the PEG on the surface; therefore, Rapa was entrapped into these particles simultaneously with the nanoparticle formation by dissolving the drug in the organic phase in the nanoprecipitation process. In this way, the drug-loaded pegylated nanoparticles (Rapa-loaded nano-PEG sample) were produced, whose mean size and $\zeta$ potential values are not affected by the presence of Rapa.

The amount of entrapped drug, reported as both drug loading (DL\%) and entrapment efficiency (EE\%), was quantified by HPLC analysis and reputed to be, respectively, 14.4 wt $\%$, and $82 \mathrm{wt} \%$.

\section{Biological assay}

Conceiving the Rapa-loaded nano-PEG for the local treatment of inflammation associated with COPD and asthma, biocompatibility has been evaluated on human bronchial epithelial cells (16-HBE). As can be seen in Fig. 3, both empty
nano-PEG and Rapa-loaded nano-PEG have no significant effects on cell viability after 24 - and 48 -h incubation, being the cell viability always higher than $80 \%$, at chosen experimental conditions. At the same time, the free drug shows a significant cytotoxicity dependent on either dose or incubation time. This result could be explained considering that, when cells are incubated with Rapa-loaded nanoparticles, the released drug concentration is lower than when incubated with free drug due to the drug release kinetics. Therefore, it can be stated that the lower toxicity could be mainly due to the lower concentration of intact drug present in the incubation medium.

\section{Development of Rapa-loaded NiM dry powders}

To turn nanoparticles into inhalable dry powders, Rapaloaded nano-PEG particles were entrapped into micrometer particles of mannitol (Man). The latter was chosen because it is a largely used pharmaceutical excipient as well as a therapeutically active and safe substance for inhalation, being able to vary the viscoelastic properties of the mucus and to increase the hydration of the periciliary fluid layer [26]. Microparticles, named $\mathrm{NiM}_{\text {(Rapa/PEG) }}$, were obtained by spray drying (SD), by following the experimental conditions and parameters described in the experimental part, with a yield of $67 \mathrm{wt} \%$. After production, the morphology and diameter were investigated by combining scanning electron microscopy (SEM) (analyzed as powder), and optical microscopy $(\mathrm{OM})$ analyses. Representative images are reported in Fig. 4.

The mean diameter of obtained $\mathrm{NiM}_{(\mathrm{Rap} / \mathrm{PEG})}$ particles was calculated from SEM, and OM images with the help of the software ImageJ were almost comparable and resulted to be, respectively, $1.53 \pm 0.26 \mu \mathrm{m}$ and $1.71 \pm 0.36 \mu \mathrm{m}$.

$\mathrm{NiM}_{\text {(Rapa/PEG) }}$ particles were dispersed in water, and the obtained nanoparticle dispersion was characterized in terms of the mean size, which resulted to be tripled compared to freshly prepared. Therefore, the powder formulation, when in contact with an aqueous medium, released empty and Rapa-loaded nano-PEG with mean size lower than $200 \mathrm{~nm}$ and $\zeta$ potential values comparable with the fresh nanoparticle dispersion. In particular, mean sizes were equal to 191.5
Table 3 XPS Surface chemical composition of obtained nanoparticles $^{\text {a }}$

\begin{tabular}{|c|c|c|c|c|c|c|}
\hline & & $\mathrm{C}-\mathrm{C} / \mathrm{C}-\mathrm{H}$ & $\mathbf{C}-\mathbf{N}$ & C-O & $\mathrm{N}-\mathrm{C}=\mathrm{O}$ & $\mathrm{OC}=\mathrm{O}$ \\
\hline Nanoparticle sample: & BE (eV) & 284.8 & 286.0 & 286.2 & 287.6 & 288.6 \\
\hline Empty PCL nano ${ }^{b}$ & & 66.6 & - & 16.6 & - & 16.8 \\
\hline Empty nano & & 57.1 & 6.3 & 16.6 & 6.6 & 13.4 \\
\hline Empty nano-PEG & & 39.1 & 4.5 & 42.9 & 4.3 & 9.2 \\
\hline
\end{tabular}

${ }^{a}$ Relative distribution of the carbon species on the nano surface determined by a curve-fitting procedure of the photoelectron peaks

${ }^{b}$ Nano obtained from PCL alone by nanoprecipitation 


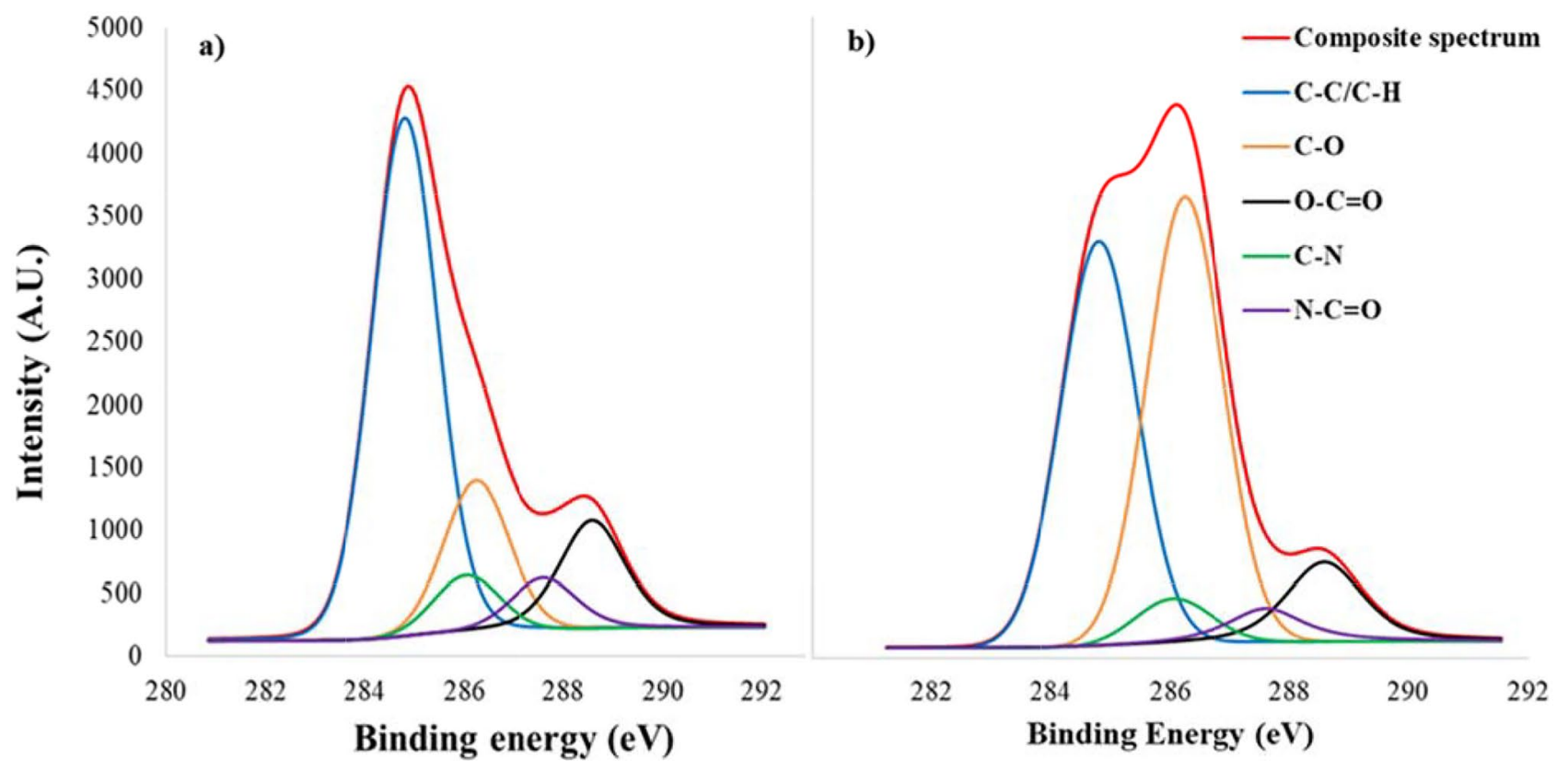

Fig. 2 Curve-fitting of the C $1 \mathrm{~s}$ spectrum of (a) empty nano and (b) empty nano-PEG samples

and $162.1 \mathrm{~nm}$, while $\zeta$ potential values equal to $-13.8 \pm 7.7$ and $-12.2 \pm 9.6, \mathrm{mV}$ respectively, for empty and drugloaded nano-PEG. Although a size increase is observed after $\mathrm{SD}$, it is acceptable considering that the mean size is still below $200 \mathrm{~nm}$, therefore potentially able to spread in the mucus [23]. Moreover, the absence of thermal degradation phenomena on both the drug and the copolymer due to the SD process was evaluated by HPLC and SEC analyses, respectively (data not shown).

Having a macrolide structure, Rapa undergoes chemical instability in physiological fluids [35]. Therefore, the entrapment into nano-PEG could protect the drug from degradation phenomena and allow a controlled release of the entrapped drug, maintaining a proper drug concentration in the administration site.

To evaluate the effect of Rapa entrapment into nano-PEG on the drug degradation, a stability study was carried out in simulated lung fluid or cell medium (Dulbecco's phosphatebuffered saline (DPBS): fetal bovine serum (FBS) (90:10 $\mathrm{v} / \mathrm{v})$ mixture) [30, 32]. The presence of FBS increases the drug solubility as well as the degradation, compared to saline medium alone [36, 37]. In particular, the free drug or a Rapa-loaded nano-PEG dispersion (obtained by dispersion of a proper $\mathrm{NiM}_{(\mathrm{Rapa} / \mathrm{PEG})}$ amount) were dispersed in
Fig. 3 16-HBE viability $\%$ after incubation in the presence of empty or Rapaloaded nano-PEG in the range $0.05-0.075 \mathrm{mg} / \mathrm{mL}$, after $24 \mathrm{~h}$ (a) and $48 \mathrm{~h} \mathrm{(b)}$

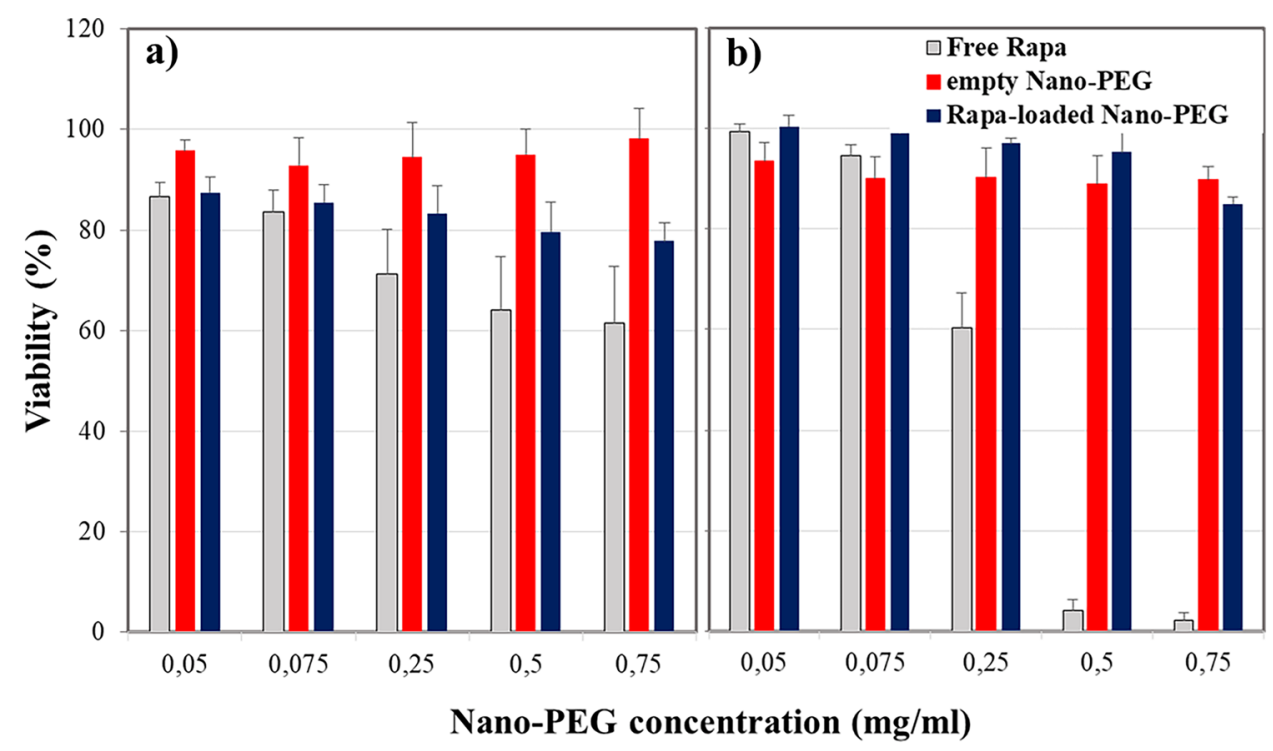


Fig. 4 SEM and OM images of $\mathrm{NiM}_{(\text {Rapa/PEG) }}$ particles. The bar represents $20 \mu \mathrm{m}$

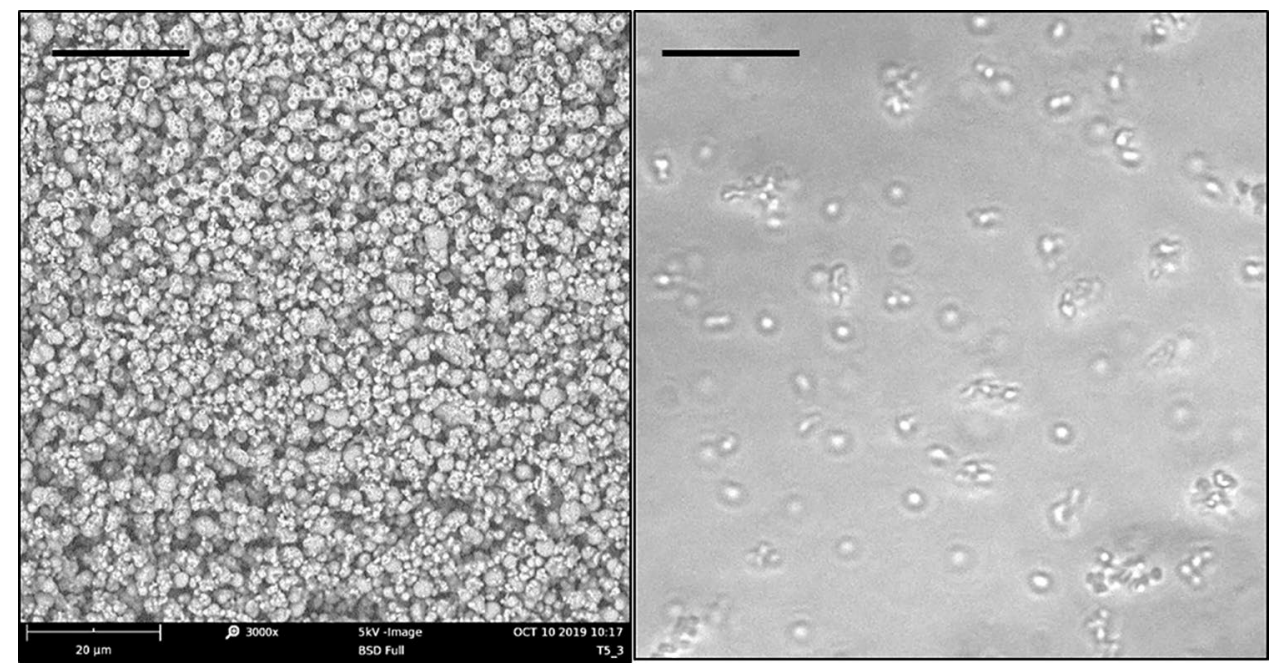

each medium, and, at fixed time intervals, the total amount of intact drug was quantified by HPLC. Results are reported in Fig. 5.

After $24 \mathrm{~h}$, the incubation of free Rapa in simulated lung fluid or cell medium resulted in a reduction of the amount of intact drug down to 40 and $25 \mathrm{wt} \%$, respectively (triangles in Fig. 5). At the same time, the amount of intact drug found in each medium upon incubation of $\mathrm{NiM}_{(\mathrm{Rap} / \mathrm{PEG})}$ was equal to 90 and $75 \mathrm{wt} \%$ of the total entrapped drug, in simulated lung fluid or cell medium respectively (squares in Fig. 5). It can be reasonably stated that Rapa loading into the nanoparticulate matrices protects it from degradation in both simulated media.

This result supports the hypothesis that the incorporation of Rapa into a carrier can improve its stability profile, which currently causes significant changes in the bioavailability of the marketed oral dosage form. Furthermore, the possibility of administering these nanoparticles by inhalation, thanks to the NiM strategy, could optimize the bioavailability of the drug into the lungs.

To evaluate the fraction of intact Rapamycin in the incubation media, on the $\mathrm{NiM}_{\text {(Rapa/PEG) }}$ sample, a drug release study was also carried out in simulated lung fluid and cell medium. In particular, the intact drug released in each incubation medium was quantified over time. Obtained data are reported in Fig. 6.

Results showed that Rapa is released from the nanoparticles in a controlled way, and that, although the total drug amount in the release medium is lowered by degradation phenomena, a constant amount of intact drug is maintained over time.

\section{Rapa-loaded NiM aerosol performance}

The main driving forces affecting the performance of a DPI are the inspiratory flow generated by the patient, and the turbulence produced inside the device, which depends on its intrinsic technical characteristics. The aerosol performance of $\mathrm{NiM}_{(\mathrm{Rapa} / \mathrm{PEG})}$ was investigated upon delivery from either a medium-resistance (TurboSpin ${ }^{\circledR}$ ) or a low-resistance (RS01) DPI. Since in the case of medium-resistance DPIs, a limited dependence of powder aerosolization on the airflow rate is expected, and aerosol performance was evaluated at 60 $\mathrm{L} / \mathrm{min}$. On the other hand, the intrinsic low-resistance of RS01 required a higher airflow rate to aerosolize the powder, that is $90 \mathrm{~L} / \mathrm{min}$. Results are shown in Fig. 7 as percentage of emitted dose deposited on the NGI cups and cumulative mass recovered as a function of the cutoff diameter. As expected, the NGI deposition pattern of NiM dry powders varied as a function of the DPI resistance. When delivered through TurboSpin ${ }^{\circledR}$ at $60 \mathrm{~L} / \mathrm{min}$ flow rate (Fig. 7A), more

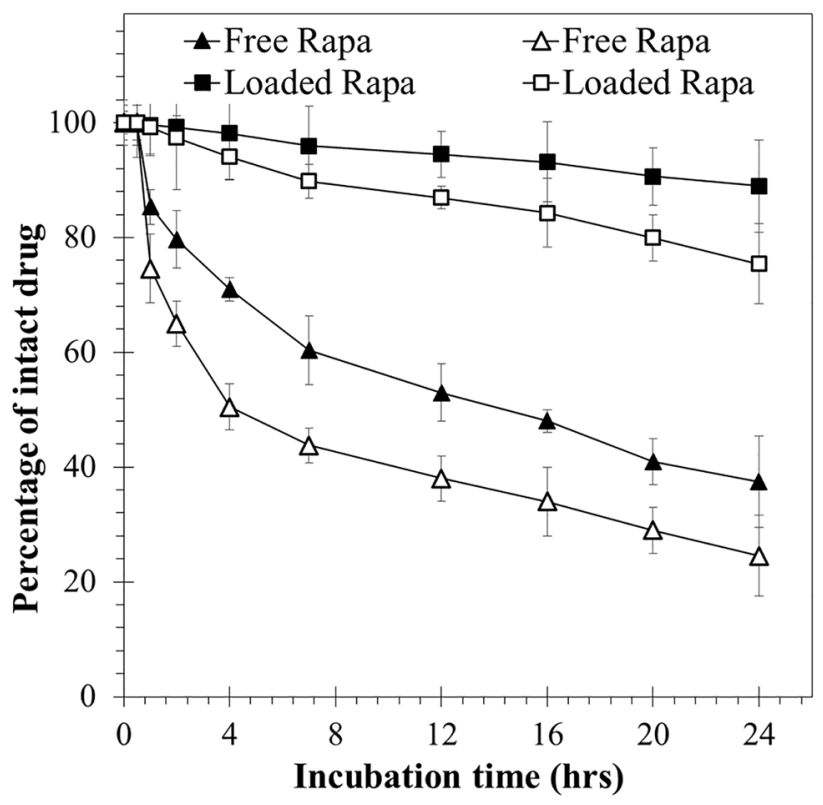

Fig. 5 Stability of free Rapa (triangle) or loaded into $\mathrm{NiM}_{(\mathrm{Rapa} / \mathrm{PEG})}$ (square), in simulated lung fluid (full indicator), and cell medium (empty indicator), at $\mathrm{pH}$ 7.4. Data represent mean $\pm \mathrm{SD}(n=3)$ 
than $70 \%$ of the dry powder sample was recovered from throat and cup 1 (MMAD $>8.06 \mu \mathrm{m})$, suggesting poor aerosolization properties. In fact, $\mathrm{NiM}_{\text {(Rapa/PEG) }}$ particles displayed an FPF (i.e., particles with an MMAD lower than $4.46 \mu \mathrm{m}$ ) of $10.6 \pm 2.8 \%$ and an RF of $16.8 \pm 2.5 \%$. Since the cumulative mass aerosolized was limited (ca. 30\%), the value of $\mathrm{MMAD}_{\exp }$ could not be determined.

The deposition pattern of the $\mathrm{NiM}_{(\mathrm{Rapa} / \mathrm{PEG})}$ dry powder improved when using RS01 (Fig. 7B). In fact, the amount of NiM found in the throat and cup 1 of the NGI lowered down to the benefit of cups 2-7 and MOC (cutoff diameter $<6.48 \mu \mathrm{m}$ ), where more than $45 \%$ of the recovered dose was deposited. In turn, an RF as high as $42.5 \pm 4.98 \%$ was calculated. The $\mathrm{MMAD}_{\text {exp }}$ was $7.09 \pm 1.25 \mu \mathrm{m}$, suggesting a good potential for bronchial deposition of the developed NiMs. Overall, results suggest that NiM is better suited to a low resistance DPI.

\section{NiM interactions with the mucus barrier}

From what has been reported so far, the followed strategy made it possible to obtain $\mathrm{NiM}_{(\mathrm{Rapa} / \mathrm{PEG})}$, consisting in pegylated Rapa-loaded nanoparticles embedded into Man microparticles. The latter, once placed in contact with fluids that mimic biological ones, can dissolve to reconstitute a dispersion of nanoparticles, capable in turn of protecting the drug. However, it must be also considered that, to reach the bronchial epithelium, these particles must spread through a

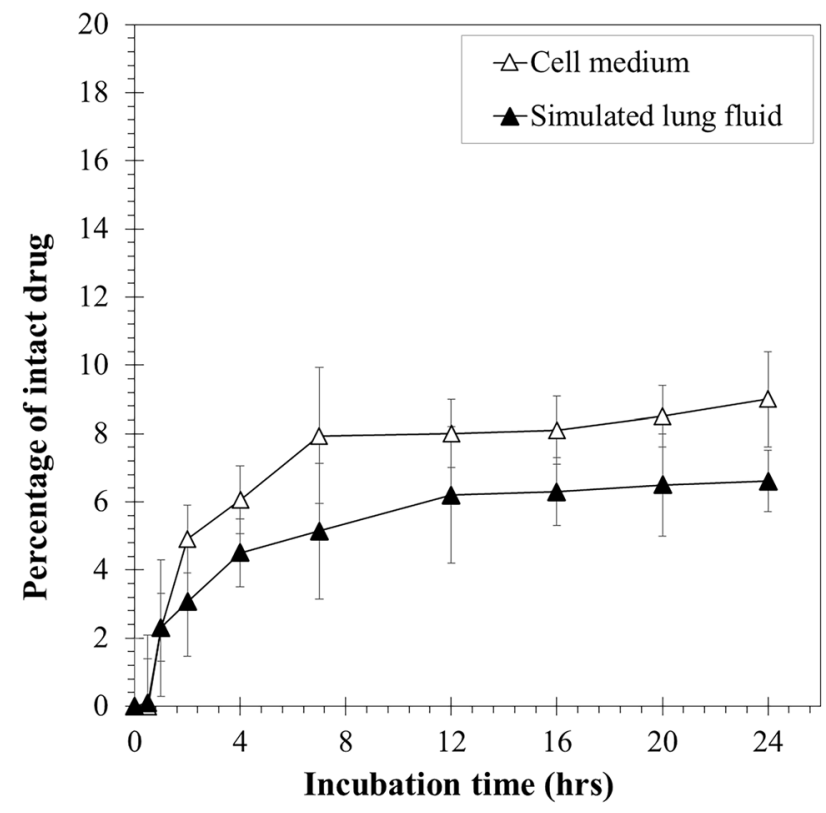

Fig. 6 Rapa release profile in simulated lung fluid (full indicator), and cell medium (empty indicator), at $\mathrm{pH}$ 7.4. Data represent mean $\pm \mathrm{SD}$ $(n=3)$ layer of mucus, which can vary in thickness and composition (i.e., in mucin concentration) according to the disease and its severity.

Therefore, to evaluate the possible interactions with the components of the mucus, which would be unfavorable for the diffusion of particles through it, rheological measurements were carried out.
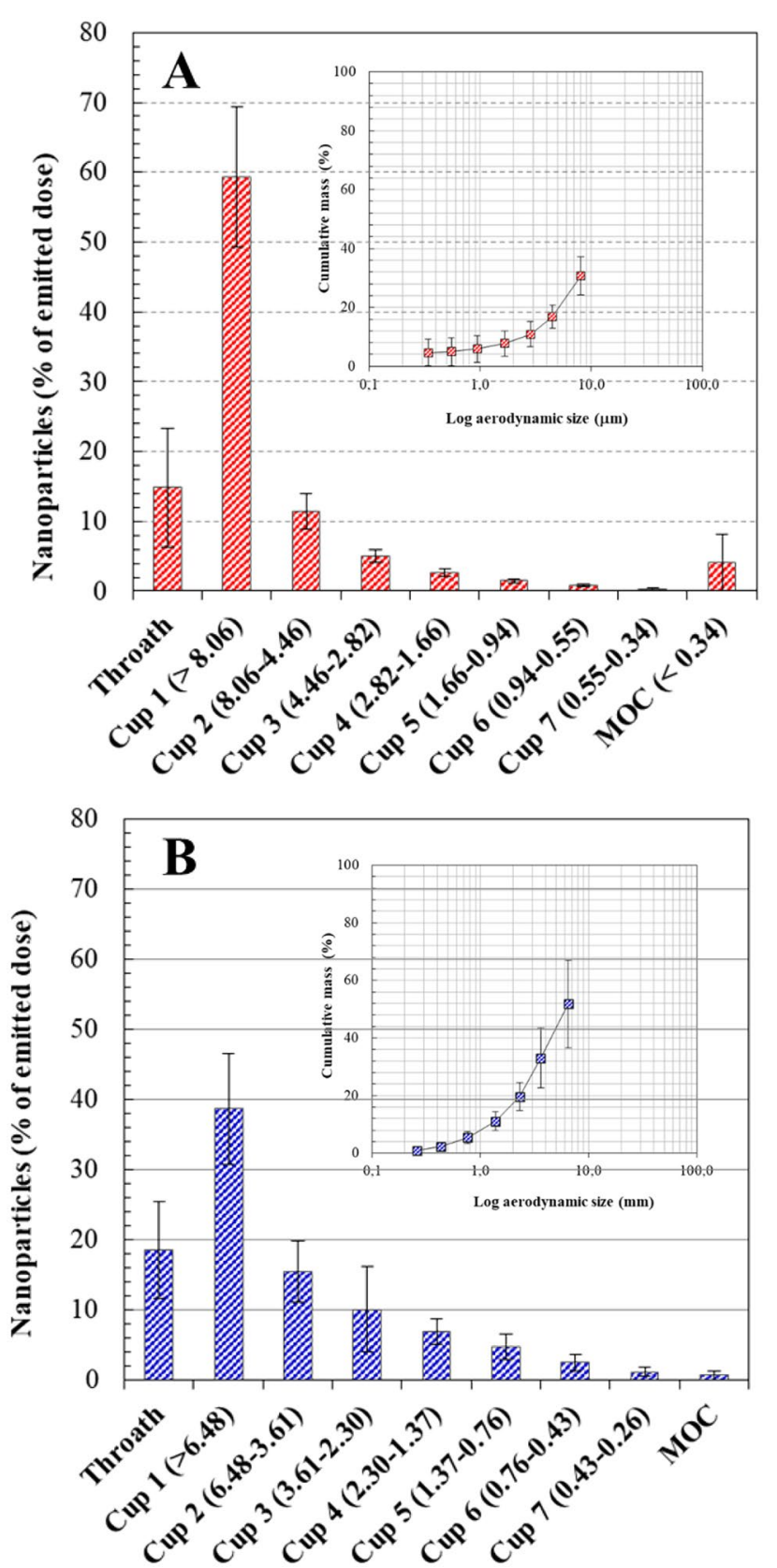

Fig. 7 In vitro aerosol performance of $\mathrm{NiM}_{(\mathrm{Rap} / \mathrm{PEG})}$ upon delivery by the breath-actuated DPIs: A TurboSpin ${ }^{\circledR}(60 \mathrm{~L} / \mathrm{min})$. B RS01 (90 L/ min). Main panels: NGI deposition patterns. Insets: cumulative mass recovered as a function of the cutoff diameter of the respective NGI stage 
In particular, $\mathrm{NiM}_{(\mathrm{Rapa} / \mathrm{PEG})}$ particles were incubated with a mucin dispersion in PBS, and the complex viscosity was measured for $30 \mathrm{~min}$. The experiment was also repeated in the presence of $\mathrm{NiM}_{(\mathrm{Rapa})}$ and in the presence of free drug (at a concentration corresponding to that carried inside $\left.\mathrm{NiM}_{(\text {Rapa/PEG) }}\right)$. Mucin alone and chitosan were used respectively as the negative and positive control. Data are reported in Fig. 8A.
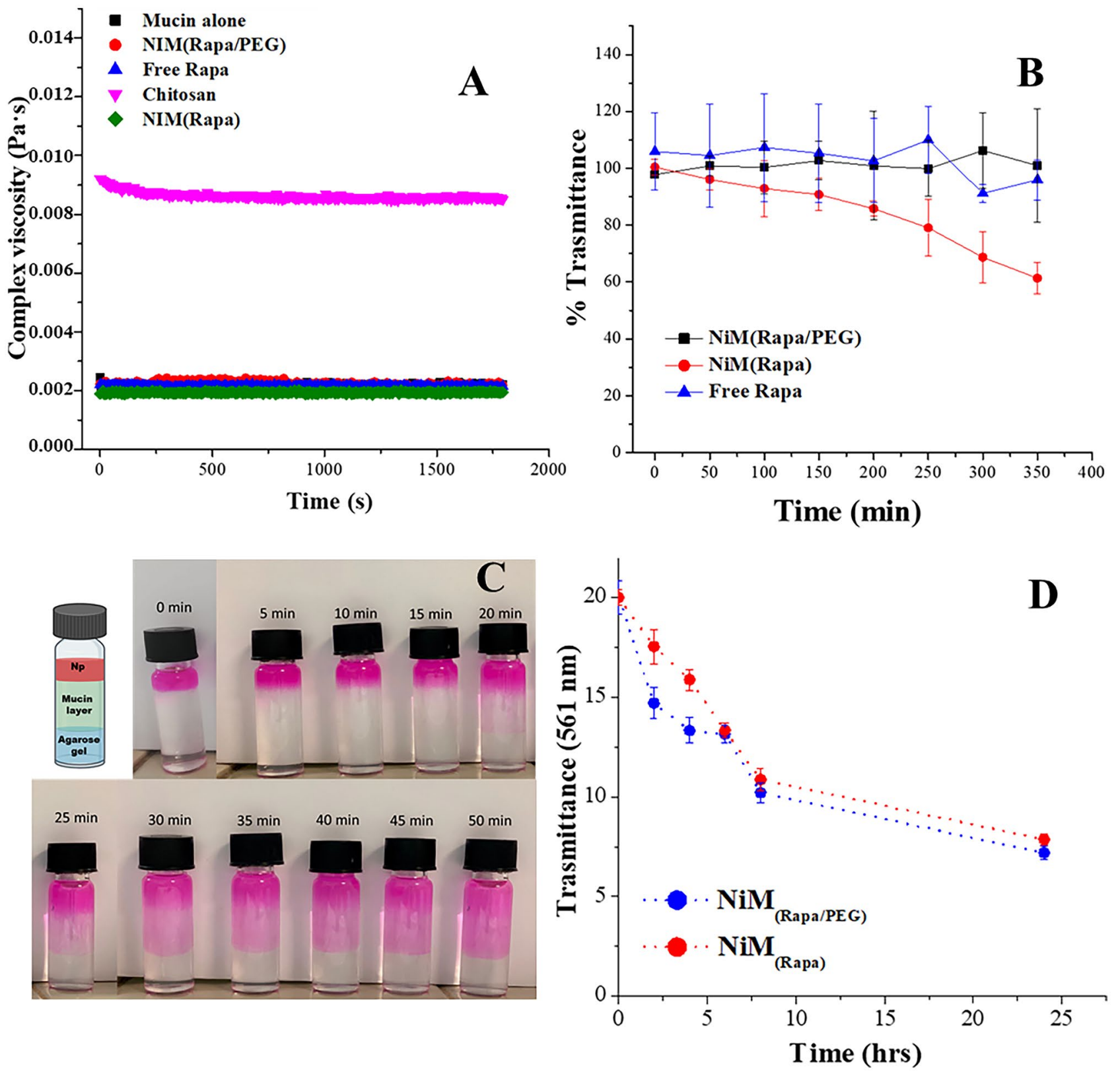

Fig. 8 A Complex viscosity $\left(\eta^{*}\right)$ of mucin dispersion treated with $\mathrm{NiM}_{\text {(Rapa/PEG) }}$ and $\mathrm{NiM}_{\text {(Rapa) }}$ particles, Rapa (at a concentration value corresponding to the drug loaded into the $\mathrm{NiM}_{(\mathrm{Rap} / \mathrm{PEG})}$, after $1 \mathrm{~h}$ incubation at $37{ }^{\circ} \mathrm{C}$. $\eta^{*}$ of untreated mucin dispersion and treated with chitosan is used as negative and positive control, respectively. $\mathbf{B}$ Turbidity values of $\mathrm{NiM}_{(\mathrm{Rapa} / \mathrm{PEG})}$, $\mathrm{NiM}_{\text {(Rapa) }}$, free drug (at a concen-

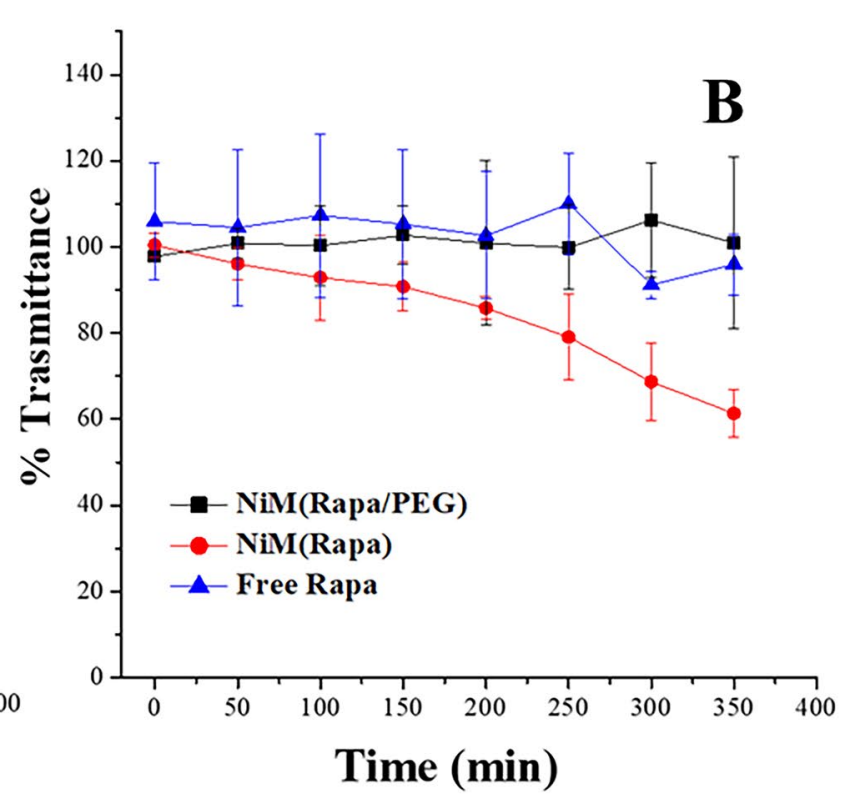

As can be seen, no significant difference was found in the rheological behavior between mucin alone and mucin containing NiM particles (pegylated or not) or free Rapa, while chitosan dispersion in mucin shows a significantly higher complex viscosity compared to all the other samples.

As well known, the interaction capacity of polymers with ments, since polymer-mucin interactions would result in a

tration lower or higher the drug solubility), incubated with a mucin dispersion $(1 \mathrm{mg} / \mathrm{mL})$. C Muco-diffusion test of Rapa-loaded nanoPEG through a mucus layer. D Transmittance at $\lambda 561 \mathrm{~nm}$ of agarose gel recovered after different incubation times with $\left.\mathrm{NiM}_{(\mathrm{Rapa} / \mathrm{PEG}}\right)$ and $\mathrm{NiM}_{(\text {Rapa })}$ mucus can also be evaluated through turbidity measure- 
reduction of sample transmittance. Therefore, transmittance measurements at $\lambda=570 \mathrm{~nm}$ were carried out on samplemucin dispersions as a function of incubation time (Fig. 8B).

As can be seen, $\mathrm{NiM}_{\text {(Rapa/PEG) }}$ particles have a low tendency to interact with mucin independently of the incubation time. On the contrary, $\mathrm{NiM}_{(\mathrm{Rapa})}$ interact with mucin as the incubation time increases, as the transmittance reduces over time. Data confirm that the surface pegylation confers to the nanoparticles the potential capability to diffuse through the mucus. Moreover, the absence of interactions of free Rapa with mucin was also demonstrated.

The capability of $\mathrm{NiM}_{\text {(Rapa/PEG) }}$ to diffuse through the mucus layer was also confirmed by a muco-diffusion assay, by exploiting the UV absorption at $\lambda$ of $561 \mathrm{~nm}$ of the RhB covalently linked to the nano-PEG. In particular, a layered system was created consisting of an agarose gel, (which acts as an acceptor compartment), and on top a mucin dispersion layer in PBS $(1 \mathrm{mg} / \mathrm{mL})$. As depicted in Fig. $8 \mathrm{C}$, the $\mathrm{NiM}_{(\mathrm{Rap} / \mathrm{PEG})}$ dispersion was placed on top of the mucin layer and subsequently, each vial was incubated at $37^{\circ} \mathrm{C}$; at defined time intervals, the mucin dispersion was removed, and the agarose gels were rinsed with distilled water, dissolved at $60^{\circ} \mathrm{C}$ and analyzed by UV spectrophotometry. The figure also shows a photograph where it is possible to see the diffusion of the particles through the mucin layer in the first $50 \mathrm{~min}$ after incubation; only after $25 \mathrm{~min}$, it is possible to observe how some particles have crossed the entire mucus layer and reached the agarose layer.

Transmittance values obtained from UV analysis, reported in Fig. 8D, showed that the amount of $\mathrm{NiM}_{(\mathrm{Rapa} / \mathrm{PEG})}$ that diffuses through the mucus layer increases as a function of the incubation time and that, in the first 5-h incubation, this amount is significantly higher than $\mathrm{NiM}_{\text {(Rapa) }}$. Moreover, for either $\mathrm{NiM}_{(\mathrm{Rapa} / \mathrm{PEG})}$ or $\mathrm{NiM}_{(\mathrm{Rapa})}$ samples, the more the incubation time increases, the more the transmittance is reduced, up to about one-third of the starting value after $24 \mathrm{~h}$.

\section{Conclusions}

In this work, we described the production of a powder formulation for the pulmonary administration of Rapamycin (Rapa), a powerful anti-inflammatory drug, whose local administration in the lungs could expand its use in therapy. In particular, the formulation was obtained by spray-drying and was made up of mannitol-based microparticles containing Rapa-loaded pegylated nanoparticles. The latter were obtained by the nanoprecipitation of the PHEA-g-RhB-g-SUCC-PCL-g-PEG graft copolymer, which was synthesized step by step from the $\alpha, \beta-\operatorname{poly}(N$ 2-hydroxyethyl)-D,L-aspartamide (PHEA) by grafting of rhodamine $\mathrm{B}(\mathrm{RhB})$, a succinylated derivative of poly- $\varepsilon$ caprolactone (PCL-SUCC), and with polyethylene glycol
(PEG). It has been shown that these microparticles possessed the aerodynamic properties suitable for administration by means of dry powder inhaler devices and that, once placed in contact with lung simulated fluids, were able to dissolve and release Rapa-loaded pegylated nanoparticles, potentially able to diffuse through the mucus. The mucusdiffusion capacity could be attributed to the presence of PEG on the surface, as showed by XPS analysis. Produced NiM were also able to release and protect Rapa in simulated lung and cell fluids.

Acknowledgements Authors thank ATeNCenter of University of Palermo - Laboratory of Preparation and Analysis of Biomaterials for scanning electron microscopy analysis, Mr. Francesco Paolo Bonomo for technical support.

Open Access This article is licensed under a Creative Commons Attribution 4.0 International License, which permits use, sharing, adaptation, distribution and reproduction in any medium or format, as long as you give appropriate credit to the original author(s) and the source, provide a link to the Creative Commons licence, and indicate if changes were made. The images or other third party material in this article are included in the article's Creative Commons licence, unless indicated otherwise in a credit line to the material. If material is not included in the article's Creative Commons licence and your intended use is not permitted by statutory regulation or exceeds the permitted use, you will need to obtain permission directly from the copyright holder. To view a copy of this licence, visit http://creativecommons.org/licenses/by/4.0/.

\section{References}

1. Racanelli AC, Kikkers SA, Choi AMK, Cloonan SM. Autophagy and inflammation in chronic respiratory disease. Autophagy. 2018;14:221-32.

2. Thakur AK, Chellappan DK, Dua K, Mehta M, Satija S, Singh I. Patented therapeutic drug delivery strategies for targeting pulmonary diseases. Expert Opin Ther Pat Taylor \& Francis. 2020;0:1.

3. Pulivendala G, Bale S, Godugu C. Inhalation of sustained release microparticles for the targeted treatment of respiratory diseases. Drug Deliv. Transl. Res. 2020;10:339-53.

4. Wang H, Wu L, Sun X. Intratracheal delivery of nano- and microparticles and hyperpolarized gases: a promising strategy for the imaging and treatment of respiratory disease. Chest Elsevier Inc. 2020;157:1579-90.

5. Beck-Broichsitter M, Merkel OM, Kissel T. Controlled pulmonary drug and gene delivery using polymeric nano-carriers. J Control Release [Internet]. Elsevier B.V.; 2012;161:214-24. Available from: https://doi.org/10.1016/j.jconrel.2011.12.004

6. Nurbaeti SN, Brillault J, Tewes F, Olivier JC. Sustained-release microparticle dry powders of chloramphenicol palmitate or thiamphenicol palmitate prodrugs for lung delivery as aerosols. Eur J Pharm Sci. 2019;138:105028.

7. Rodrigues TC, Oliveira MLS, Soares-Schanoski A, ChavezRico SL, Figueiredo DB, Gonçalves VM, et al. Mucosal immunization with PspA (pneumococcal surface protein A)-adsorbed nanoparticles targeting the lungs for protection against pneumococcal infection. PLoS ONE. 2018;13:1-17.

8. Sarcinelli MA, Martins da Silva T, Artico Silva AD, Ferreira de Carvalho Patricio B, Mendes de Paiva FC, Santos de Lima 
$\mathrm{R}$, et al. The pulmonary route as a way to drug repositioning in COVID-19 therapy. J Drug Deliv Sci Technol. 2021;63.

9. Newman SP. Delivering drugs to the lungs: the history of repurposing in the treatment of respiratory diseases. Adv. Drug Deliv. Rev. [Internet]. Elsevier B.V.; 2018;133:5-18. Available from: https://doi.org/10.1016/j.addr.2018.04.010

10. Mehta PP, Dhapte-Pawar VS. Repurposing drug molecules for new pulmonary therapeutic interventions. Drug Deliv. Transl. Res. 2020

11. Laplante M, Sabatini DM. MTOR signaling in growth control and disease. Cell. 2012;149:274-93.

12. Mitani A, Ito K, Vuppusetty C, Barnes PJ, Mercado N. Restoration of corticosteroid sensitivity in chronic obstructive pulmonary disease by inhibition of mammalian target of rapamycin. Am J Respir Crit Care Med. 2016;193:143-53.

13. He Y, Zuo C, Jia D, Bai P, Kong D, Chen D, et al. Loss of DP1 aggravates vascular remodeling in pulmonary arterial hypertension via mTORC1 signaling. Am J Respir Crit Care Med. 2020;201:1263-76.

14. Mushaben EM, Brandt EB, Hershey GKK, Le Cras TD. Differential effects of rapamycin and dexamethasone in mouse models of established allergic asthma. PLoS One. 2013;8.

15. Omarjee L, Perrot F, Meilhac O, Mahe G, Bousquet G, Janin A. Immunometabolism at the cornerstone of inflammaging, immunosenescence, and autoimmunity in COVID-19. Aging (Albany. NY). 2020;12:1-16.

16. Husain A, Byrareddy SN. Rapamycin as a potential repurpose drug candidate for the treatment of COVID-19. Chem. Biol. Interact. [Internet]. Elsevier B.V. 2020;331:109282. Available from: https://doi.org/10.1016/j.cbi.2020.109282

17. Haeri A, Osouli M, Bayat F, Alavi S, Dadashzadeh S. Nanomedicine approaches for sirolimus delivery: a review of pharmaceutical properties and preclinical studies. Artif Cells, Nanomedicine Biotechnol. 2018;46:1-14.

18. Carvalho SR, Watts AB, Peters JI, Liu S, Hengsawas S, EscotetEspinoza MS, et al. Characterization and pharmacokinetic analysis of crystalline versus amorphous rapamycin dry powder via pulmonary administration in rats. Eur J Pharm Biopharm. 2014;88:136-47.

19. Landh E, Moir LM, Gomes Dos Reis L, Traini D, Young PM, Ong HX. Inhaled rapamycin solid lipid nano particles for the treatment of Lymphangioleiomyomatosis. Eur J Pharm Sci. [Internet]. Elsevier. 2020;142:105098. Available from: https:// doi.org/10.1016/j.ejps.2019.105098

20. Alexis F, Pridgen E, Molnar LK, Farokhzad OC. Factors affecting the clearance and biodistribution of polymeric nanoparticles. Mol Pharm American Chemical Society. 2008;5:505-15.

21. Pilcer G, Amighi K. Formulation strategy and use of excipients in pulmonary drug delivery. Int J Pharm. 2010;392:1-19.

22. Huckaby JT, Lai SK. PEGylation for enhancing nanoparticle diffusion in mucus. Adv Drug Deliv Rev. [Internet]. Elsevier B.V. 2018;124:125-39. Available from: https://doi.org/10. 1016/j.addr.2017.08.010

23. Suk JS, Xu Q, Kim N, Hanes J, Ensign LM. PEGylation as a strategy for improving nanoparticle-based drug and gene delivery. Adv. Drug Deliv. Rev. [Internet]. Elsevier B.V. 2016;99:2851. Available from: https://doi.org/10.1016/j.addr.2015.09.012

24. Lechanteur A, Evrard B. Influence of composition and spraydrying process parameters on carrier-free DPI properties and behaviors in the lung: a review. Pharmaceutics. 2020;12.
25. Elsayed MMA. Microstructural characterization of carrier-based dry powder inhalation excipients: insights and guidance. Int J Pharm. [Internet]. Elsevier. 2019;568:118482. Available from: https://doi.org/10.1016/j.ijpharm.2019.118482

26. Porsio B, Craparo EF, Mauro N, Giammona G, Cavallaro G. Mucus and cell-penetrating nanoparticles embedded in nanointo micro formulations for pulmonary delivery of ivacaftor in patients with cystic fibrosis. ACS Appl Mater Interfaces. 2018;10:165-81.

27. Craparo EF, Drago SE, Giammona G, Cavallaro G. Production of polymeric micro- and nanostructures with tunable properties as pharmaceutical delivery systems. Polymer (Guildf). 2020;200:122596.

28. Giammona G, Pitarresi G, Craparo EF, Cavallaro G, Buscemi S. New biodegradable hydrogels based on a photo-cross-linkable polyaspartamide and poly(ethylene glycol) derivatives. Release studies of an anticancer drug. Colloid Polym Sci. 2001;279:771-83.

29. Mendichi R, Giammona G, Cavallaro G, Giacometti SA. Molecular characterization of $\alpha, \beta$-poly [(N-hydroxyethyl)-DLaspartamide] by light scattering and viscometry studies. Polymer (Guildf). 2000;41:8649-57.

30. Craparo EF, Cabibbo M, Conigliaro A, Barreca MM, Musumeci $\mathrm{T}$, Giammona $\mathrm{G}$, et al. Rapamycin-loaded polymeric nanoparticles as an advanced formulation for macrophage targeting in atherosclerosis. 2021

31. Craparo EF, Porsio B, Mauro N, Giammona G, Cavallaro G. Polyaspartamide-polylactide graft copolymers with tunable properties for the realization of fluorescent nanoparticles for imaging. Macromol Rapid Commun. 2015;36:1409-15.

32. Marques MRC, Loebenberg R, Almukainzi M. Simulated biologic fluids with possible application in dissolution testing. Dissolution Technol. 2011;15-28.

33. Pitarresi G, Palumbo FS, Calabrese R, Craparo EF, Giammona G. Crosslinked hyaluronan with a protein-like polymer: novel bioresorbable films for biomedical applications. J Biomed Mater Res - Part A. 2008;84:413-24.

34. Craparo EF, Licciardi M, Conigliaro A, Palumbo FS, Giammona G, Alessandro R, et al. Hepatocyte-targeted fluorescent nanoparticles based on a polyaspartamide for potential theranostic applications. Polymer (Guildf). Elsevier Ltd. 2015;70:257-70.

35. Campos MST, Fialho SL, Pereira BG, Yoshida MI, Oliveira MA. Kinetics studies of the degradation of sirolimus in solid state and in liquid medium. J Therm Anal Calorim. 2017;130:1653-61.

36. Zhao J, Weng G, Li J, Zhu J, Zhao J. Polyester-based nanoparticles for nucleic acid delivery. Mater Sci Eng C [Internet]. Elsevier. 2018;92:983-94. Available from: https://doi.org/10.1016/j. msec.2018.07.027

37. Linares-Alba MA, Gómez-Guajardo MB, Fonzar JF, Brooks DE, García-Sánchez GA, Bernad-Bernad MJ. Preformulation studies of a liposomal formulation containing sirolimus for the treatment of dry eye disease. J Ocul Pharmacol Ther. 2016;32:11-22.

Publisher's Note Springer Nature remains neutral with regard to jurisdictional claims in published maps and institutional affiliations. 